\title{
Estudio epidemiológico del suicidio en Sevilla en 2004.
}

Epidemiological study of suicide in Seville in 2004.

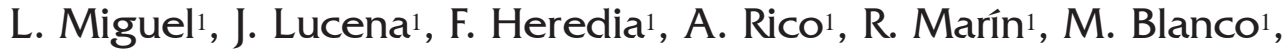 \\ E. Barrero' ${ }^{1}, M T$. Soriano y MP. Giménez ${ }^{2}$
}

\section{RESUMEN}

En este artículo se realiza un estudio epidemiológico descriptivo de las muertes de etiología suicida ocurridas en Sevilla en el año 2004 y los datos obtenidos se comparan con otros estudios previos realizados en la misma zona. La tasa de suicidio fue de $\mathbf{8 , 3 6}$ por 100.000 habitantes con una relación hombre/mujer de 2,6:I. La edad media global ha sido de 54,3 años (52,7 años en varones y 58,7 años en mujeres). El mayor número de suicidios $(58,5 \%)$ se producen en el segundo y tercer trimestre del año (primavera y verano). En cuanto al estado civil, la mayor frecuencia se produce en personas casadas $(31,2 \%)$. Solo en el $9,5 \%$ de los suicidas existían antecedentes de intentos autolíticos previos. La ahorcadura ha sido el mecanismo más empleado en los varones $(50,9 \%)$ mientras que en las mujeres el mecanismo más frecuen-

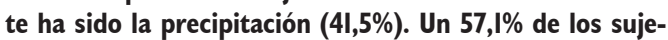
tos tenía antecedentes psiquiátricos siendo los trastornos más frecuentes la depresión o los síntomas depresi$\operatorname{vos}(65,5 \%)$ y la esquizofrenia (15,5\%). Se efectuaron análisis químico-toxicológicos en el $\mathbf{7 8 , 2} \%$ de los casos de los cuales fueron positivos el $69,6 \%$. El alcohol etílico fue la sustancia más detectada $(24,3 \%)$, seguido de las benzodiazepinas $(18,3 \%$ ) y los antidepresivos (II,3\%). Se observa una cierta estabilización en la tasa de suicidios en Sevilla desde mediados del siglo XX hasta la actualidad.

Palabras clave: Suicidio, epidemiología, Sevilla, autopsia, medicina forense.

Cuad Med Forense 2005; 11(39):43-53

\section{ABSTRACT}

In this paper, we present an epidemiological descriptive study of the suicides occurred in Seville in 2004 and the results are compared with data obtained in previous studies in the same area. Suicide rate was 8,36 per 100.000 inhabitants with a male/female ratio of 2,6:1. The medium age has been 54,3 years $(52,7$ years in males and 58,7 years in females). Suicides predominate in the second and third trimester of the year (spring and summer). Taking into account the civil status, the mayor frequency appears in married $(31,2 \%)$. Only $9,5 \%$ of the cases had antecedents of previous autolysis intents. Hanging was the most frequent mechanism to commit suicide in men (50.9\%) while in women was jumping $(41,5 \%)$. In $57,1 \%$ of cases there were antecedents of mental disorder mainly depression of depressive symptoms $(65,5 \%)$ followed by schizophrenia (15,5\%). Toxicological analyses were performed in $78,2 \%$ of cases with positive results in $69,6 \%$. Ethanol was the toxic most detected $(24,3 \%)$ followed by benzodiazepines (18,3\%) and antidepressants (1 1,3\%). According to the results of the study, we observe a certain stabilization of the suicide rates in Seville from the middle of the XXth century up to now.

Key words: Suicide, epidemiology, Seville, autopsy, forensic medicine.

Correspondencia: L. Miguel Alhambra. Servicio de Patología Forense. IML de Sevilla. Avda. Sánchez Pizjuán s/n. 41071 Sevilla. Tel. 954370644, Fax 954906834. E-mail: iaf.sevilla@andaluciajunta.es.

1 Servicio de Patología Forense. Instituto de Medicina Legal de Sevilla.

1 Servicio de Química. Instituto Nacional de Toxicología y Ciencias Forenses. Departamento de Sevilla. 


\section{INTRODUCCIÓN:}

Según la Organización Mundial de la Salud y la Organización Panamericana de la Salud (OMS/OPS), el fenómeno del suicidio es responsable de más muertes al año que las producidas anualmente por el conjunto de todos los conflictos bélicos que asolan el planeta constituyéndose en una de las principales causas de muerte en el mundo. Estas organizaciones estimaron que en el año 2000 se produjo una muerte por suicidio cada 40 segundos y un intento de suicidio cada 3 [I]. En España, de acuerdo con los datos publicados por el Instituto Nacional de Estadística, durante el año 2003 el número de suicidios consumados y tentativas ascendió a 2760 [2] (*).

A la vista de estos datos, parece evidente que el suicidio supone un problema de salud pública importante porque se trata de muertes ante las cuales se pueden adoptar medidas de prevención considerándose desde este punto de vista como muertes evitables.

En este artículo se realiza un estudio epidemiológico descriptivo de las muertes de etiología suicida ocurridas en Sevilla en el año 2004 y se comparan los datos obtenidos con estudios previos. El objetivo es conocer la tendencia de la mortalidad por suicidio a lo largo del tiempo en este área geográfica del sur de España.

\section{MATERIAL Y MÉTODOS:}

Las fuentes utilizadas para la realización de este estudio han sido los libros de registro del Servicio de Patología Forense del Instituto de Medicina Legal de Sevilla (SPF-IMLSE) donde están centralizadas todas las autopsias de Sevilla, capital y provincia. En ellos se recogen los datos de filiación del cadáver, procedencia, antecedentes patológicos, data de la muerte, causa, mecanismo y etiología de la muerte y análisis practicados. Para completar esta información se han consultado los protocolos de levantamiento del cadáver, los informes de autopsia y los resultados de los análisis químico-toxicológicos realizados en el Departamento de Sevilla del Instituto Nacional de Toxicología y Ciencias Forenses (INTCF)(**).

Para el tratamiento estadístico de los datos se ha utilizado el programa informático SPSS versión 12.0 .

\section{RESULTADOS:}

\section{I.- Frecuencia y tasas de suicido}

Durante el año 2004 se realizaron en el SPF-IMLSE 919 autopsias de las cuales 542 (59\%) correspondieron a muertes violentas y 377 (41\%) a muertes naturales. Se registraron 147 casos de suicidio de los cuales $82(55,8 \%)$ se produjeron en Sevilla capital y $65(44,2 \%)$ en el resto de la provincia. Los suicidios constituyeron el I5,9\% del total de las autopsias practicadas y el 27, 12\% de las muertes violentas.

La determinación de la tasa de suicidio se ha realizado a partir los datos del censo de población facilitados por el Instituto Nacional de Estadística y el Excmo. Ayuntamiento de Sevilla ( I de enero de 2002) siendo ésta de 8,36 por 100.000 habitantes (I I,6 y 6,2 por 100.000 habitantes en la capital y provincia respectivamente).

\section{2.- Mecanismo}

Los mecanismos empleados y sus frecuencias absolutas y en función del sexo están recogidos en la tabla $\mathrm{I}$.

\footnotetext{
${ }^{*}$ () Debido a la enorme dificultad que existe para el diagnóstico y el registro de los intentos de autolisis, consideramos que la estadística del INE refleja fundamentalmente el número de los suicidios consumados. Entendemos que los datos referentes a los intentos de autolisis son mucho mayores que los expuestos.

(**) Hasta el año 2004, los datos obtenidos de los libros de registro de autopsias realizadas en el actual SPF-IMLSE se refieren, fundamentalmente, a casos del partido judicial de Sevilla
} 
TABLA I

\begin{tabular}{|c|c|c|c|c|}
\hline MECANISMO & No DE CASOS & $\%$ & $\begin{array}{c}\mathbf{N}^{\circ} \text { DE CASOS } \\
\text { EN VARONES }\end{array}$ & $\begin{array}{c}\mathbf{N}^{\circ} \text { DE CASOS } \\
\text { EN MUJERES }\end{array}$ \\
\hline AHORCADURA & 63 & 42,9 & & \\
PRECIPITACIÓN & 35 & 23,8 & 18 & 17 \\
ARMA DE FUEGO & 11 & 7,5 & 10 & 1 \\
INTOXICACIÓN & 10 & 6,7 & 6 & 4 \\
INGESTA CÁUSTICOS & 8 & 5,4 & 3 & 5 \\
SUMERSIÓN & 7 & 4,8 & 5 & 2 \\
A LO BONZO & 6 & 4,1 & 5 & 1 \\
ARMA BLANCA & 4 & 2,7 & 3 & 1 \\
ATROPELLO TREN & 2 & 1,4 & 2 & 0 \\
SOFOCACIÓN & 1 & 0,7 & 0 & 1 \\
\hline TOTAL & \multirow{2}{*}{$\mathbf{1 4 7}$} & \multirow{2}{*}{$\mathbf{1 0 0}$} & $\mathbf{1 0 6}$ & \multirow{2}{*}{} \\
\hline
\end{tabular}

En términos generales, los mecanismos más frecuentemente empleados han sido con diferencia la ahorcadura (42,9\%) y la precipitación (23,8 \%), seguidos del arma de fuego (7,5\%) y la intoxicación medicamentosa (6,8 \%).

Si tenemos en consideración el mecanismo en función del sexo, en los varones el más empleado fue la ahorcadura (54 frente a 9 casos de ahorcadura en mujeres) seguido de la precipitación ( 8 casos frente a los 17 casos de precipitación en mujeres). El 85,7\% de las ahorcaduras fueron efectuadas por sujetos varones. Las diferencias por sexos en el caso de la precipitación no son tan llamativas siendo varones el 51,4\% y mujeres el 48,6\%.

El uso de arma de fuego es casi exclusivo del sexo masculino pues 10 de los I I casos de suicidio registrados fueron realizados por varones. Del mismo modo, de los 6 casos en los que se empleó la quemadura a lo bonzo 5 fueron varones (el 83,3\%)

En la tabla II se pone en relación el área geográfica en la que se ha producido la muerte y el mecanismo de suicidio:

TABLA II

\begin{tabular}{|c|c|c|}
\hline \multirow{2}{*}{ MECANISMO } & \multicolumn{2}{|c|}{ ÁREA } \\
\cline { 2 - 3 } & SEVILLA & PROVINCIA \\
\hline AHORCADURA & 19 & 44 \\
PRECIPITACIÓN & 25 & 10 \\
ARMA DE FUEGO & 6 & 5 \\
INTOXICACIÓN & 8 & 2 \\
INGESTA CÁUSTICOS & 8 & 0 \\
SUMERSIÓN & 6 & 1 \\
A LO BONZO & 5 & 1 \\
ARMA BLANCA & 3 & 1 \\
ATROPELLO TREN & 1 & 1 \\
SOFOCACIÓN & 1 & 0 \\
\hline TOTAL & $\mathbf{8 2}$ & $\mathbf{6 5}$ \\
\hline
\end{tabular}


El 7I,4 \% de las precipitaciones se efectuaron en Sevilla capital y el 69,8\% de las ahorcaduras en la provincia, aunque estos datos hay que interpretarlos con prudencia porque los casos en que la muerte sobrevino en el hospital, por existir un tiempo de supervivencia, se han consignado como acaecidos en la capital aun siendo posible que el acto suicida se realizara en la provincia.

\section{3.- Edad y sexo}

106 casos $(72,1 \%)$ de suicidio fueron llevados a cabo por hombres y 4 I $(27,9 \%)$ por mujeres con una relación hombre/mujer de 2,6:l. En cuanto a la edad, la máxima frecuencia se alcanza en la cuarta y quinta década de la vida, en los grupos de edad de 40-49 años (19\%) y de 50-59 años ( 19\%). La media global de edad es de 54,3 años. La edad media en varones es de 52,7 años y en mujeres de 58,7 años. Si se ponen en relación la edad y el sexo de los suicidas, en el caso de los hombres, la máxima frecuencia continúa siendo de los 40-59 años, 20,8\% de los 40-49 años y $18,9 \%$ de los 50-59 años. No obstante, en las mujeres el $22 \%$ (9 casos) de los suicidios tienen lugar en la séptima década de la vida y el 19,5\% (8 casos) en la quinta (Gráfico I).

\section{GRÁFICO 1}

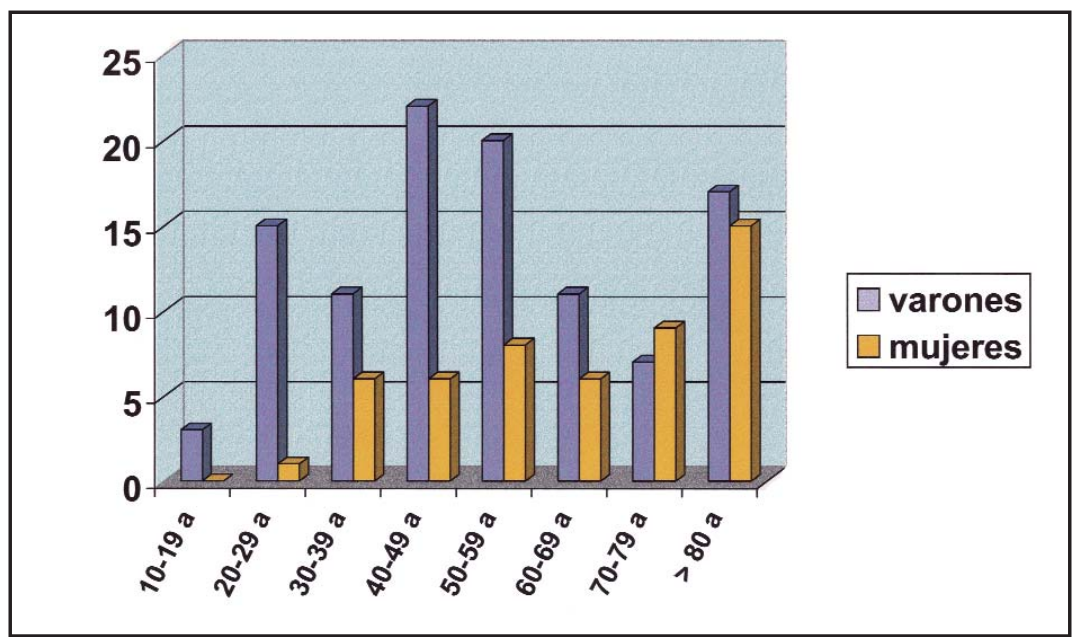

\section{4.- Lugar de procedencia del cadáver}

El 47,6\% (70 casos) de los levantamientos se efectuaron en el domicilio y el $26,5 \%$ (39 casos) en vía pública. En un 16,3\% de los casos el cadáver procedía del hospital y el resto (el 9,5\%) de otras instituciones (residencia psiquiátrica, prisión, centro de trabajo) (Gráfico 2).

\section{GRÁFICO 2}

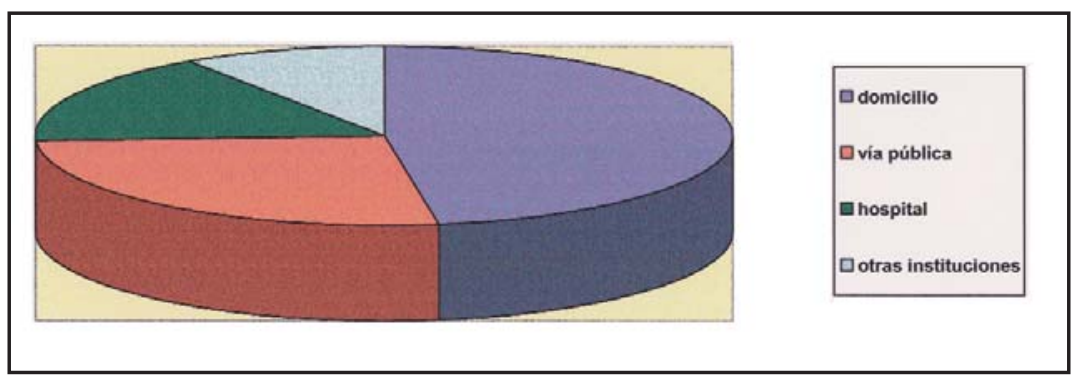




\section{5- Estado civil y sexo}

El $31,2 \%$ (46 casos) de los suicidas estaban casados, el 26,5\% (39 casos) solteros, el I4,3\% ( 2 I casos) eran viudos y un 6, I \% (9 casos) estaban separados o divorciados. En el $21,8 \%$ (32 casos) no se pudo disponer de este dato. En el caso de los hombres 29 (27,4\%) estaban solteros, 34 (32, I\%) casados, $15(14,1 \%)$ eran viudos y $8(7,5 \%)$ estaban separados o divorciados. De las mujeres $10(24,4 \%)$ eran solteras, I2 (29,3\%) casadas, 6 (I4,6\%) viudas y I (2,4\%) separadas o divorciadas (Gráfico 3).

\section{GRÁFICO 3}

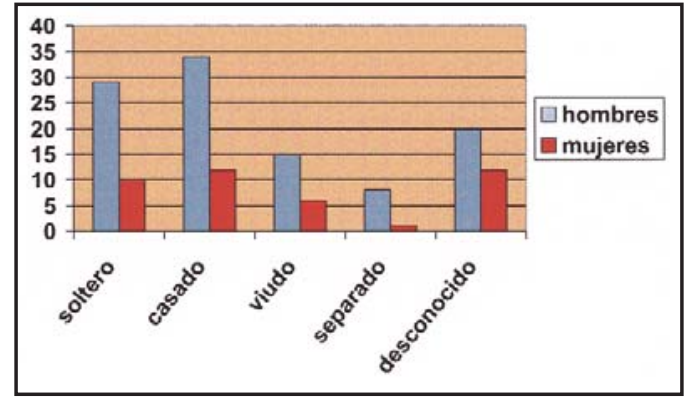

\section{6- Día de la semana y estación del año}

El 58,5\% de los suicidios se produjeron en primavera (44 casos) y verano (42 casos). El número máximo de suicidios tuvo lugar en lunes (30 casos) y el mínimo se registró en martes y jueves ( 16 casos cada día).

\section{7- Intentos previos}

En el 82,3\% ( 121 casos) no se tiene conocimiento de la existencia de intentos previos bien porque no han existido o porque se desconoce este dato. El 8,2 \% de los suicidas ( 12 casos) manifestaba ideación autolítica y el 9,5\% ( 4 casos) había realizado intentos de suicidio previamente.

\section{8-Documentos de despedida}

Solo 13 suicidas $(8,84 \%)$ dejaron notas o cartas de despedida.

\section{9- Análisis químico-toxicológicos}

Se tomaron muestras para análisis de tóxicos en 1 I 5 casos (78,2\%). Los resultados fueron negativos en 35 (30,4\%). En los 80 casos restantes (69,6\%) los resultados analíticos fueron positivos. (Gráfico 4).

\section{GRÁFICO 4}

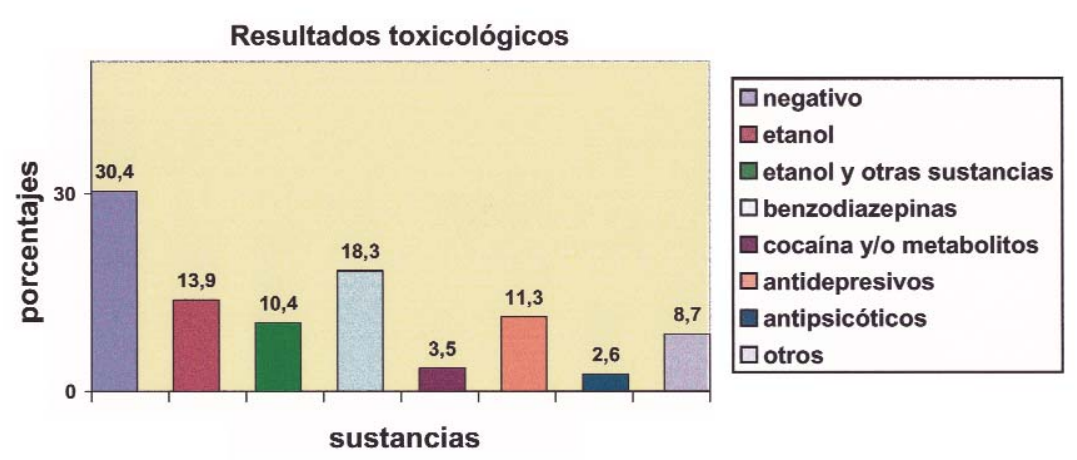


El alcohol etílico se detectó en un total de 28 casos (24,3\%), de forma aislada en el I3,9\% ( 16 casos) y combinado con otras sustancias en el 10,43\% ( 12 casos). La combinación alcohol etílico y benzodiacepinas se observó en 7 casos, de los cuales 3 fueron también positivos para antidepresivos. La combinación alcohol-cocaína y/o metabolitos se dio en 4 casos. Se obtuvieron resultados positivos de forma aislada para benzodiacepinas en el 18,3\% (21 casos) y para cocaína y/o metabolitos en 3,5\% (4 casos). En 13 casos ( 11 ,3\%) se detectaron antidepresivos y en 3 (2,6\%) antipsicóticos. En 10 casos (8,7\%) se encontraron otras sustancias (anticomiciales, antihipertensivos, fármacos utilizados en terapia intensiva, etc.).

\section{0- Antecedentes patológicos}

El 57, I \% (84 casos) de los sujetos tenía antecedentes psiquiátricos y el 7,5 \% ( I I casos) padecía enfermedad orgánica. De las personas con patología psiquiátrica, el 65,5\% (55 casos) padecía depresión o síntomas depresivos. Un 15,5\% ( 13 casos) estaba diagnosticado de esquizofrenia; en 9 casos (10,7\%) existían antecedentes de consumo de drogas de abuso y 7 personas (8,3\%) padecía otro tipo de patología. En un 35,4\% (52 casos) no se tuvo conocimiento de la existencia de antecedentes patológicos.

\section{DISCUSIÓN:}

Las tasas de suicidio ( $n^{\circ}$ de suicidios por 100.000 habitantes) permiten clasificar a los distintos países en tres grupos. Se distinguen así (Fig. I):

- Países con tasas de suicido por encima de 13

- Países con tasas de suicidio entre 6,5-13

- Países con tasas de suicidio menores de 6,5

Entre 1985 y 1994, España se encontraba situada en el tercer grupo al registrarse tasas de suicidio de 5,9 [3]. No obstante, en los últimos años ha habido un aumento en el número de suicidios en nuestro país lo que ha determinado su inclusión dentro de la segunda categoría el año 2002.

Centrándonos en Sevilla, la tasa promedio de suicidios de 1953 a 1977 fue de 6,22 siendo máxima para el quinquenio de 1958-62 (8, I y mínima para el quinquenio de 1973-77 (5,2). De 1978 a 1987 la tasa promedio fue de 7,59 con valores máximos para el año 1986 ( I I, I y y mínimos para $1979(4,5)$. En 1989 la tasa de suicidio fue de |3,6| [4,5,6,7].

En 2004 la tasa de suicidio ha sido de 8,36 por 100000 habitantes cifra que se encuentra por encima de la media de los años anteriores.

Los datos obtenidos para el 2004 no contradicen el aumento en la mortalidad por suicidio registrado en los años anteriores pero sí podría indicar cierta estabilización en las cifras. En el gráfico 5 se muestra la evolución de las tasas de suicidio en la ciudad de Sevilla desde 1970.

Es muy llamativa la evolución del porcentaje de suicidios con respecto al número de autopsias realizadas por muertes violentas. Los primeros estudios epidemiológicos del suicidio en Sevilla se remontan a 1953 por lo que es a partir de esta fecha que vamos a realizar los estudios comparativos. De 1953 a 1977 se practicaron un total de 6.762 autopsias de las cuales el 79,5\% fueron de etiología violenta. Los suicidios representaron un I4,8\% de las muertes violentas y el II,8\% de las autopsias totales.

De 1978 a 1987 se practicaron 4076 autopsias de las cuales un 73,6\% correspondieron a muertes violentas. Los suicidios representaron un $16,82 \%$ del total de muertes violentas y un $12,38 \%$ del total de autopsias [5]. 


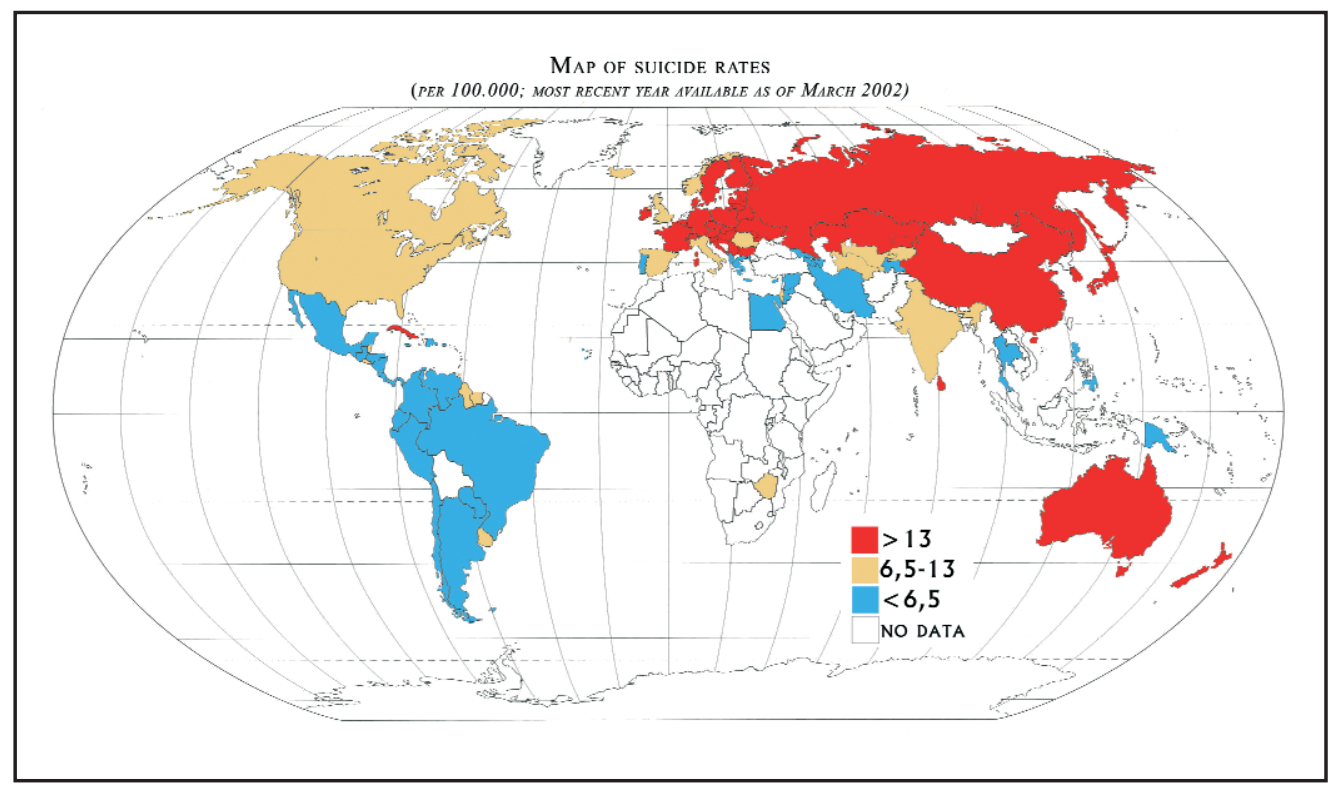

Figura 1. Mapa de tasas de suicidio.

(www.who.int/mental_health/prevention/suicide/suicideprevent/en/)

De las 919 autopsias realizadas en el SPF-IMLSE en 2004, el 59\% correspondieron a muertes violentas. Los suicidios han supuesto el 27, I $2 \%$ de las muertes violentas y el I5,9\% del total de autopsias realizadas. Estos datos ponen de manifiesto que, desde los años 1950, los suicidios han pasado de un 14,8\% de las muertes violentas hasta el 27, 12\% que se ha registrado en 2004.

La relación hombre/mujer en el suicidio no ha sufrido cambios importantes, manteniéndose relativamente estable, pese al incremento en el número de mujeres suicidas registradas de 1978 a 1987. Así, en los periodos de tiempo a que nos estamos refiriendo (1953- 1977 y 1979- I 987) se ha registrado una relación hombre/mujer de 2,5:1 y 1,67:I, respectivamente. En 1989 la relación fue de 2,2:1 y en 2004 de 2,6:1.

GRÁFICO 5

Evolución de las tasas de suicidio desde 1970-2004

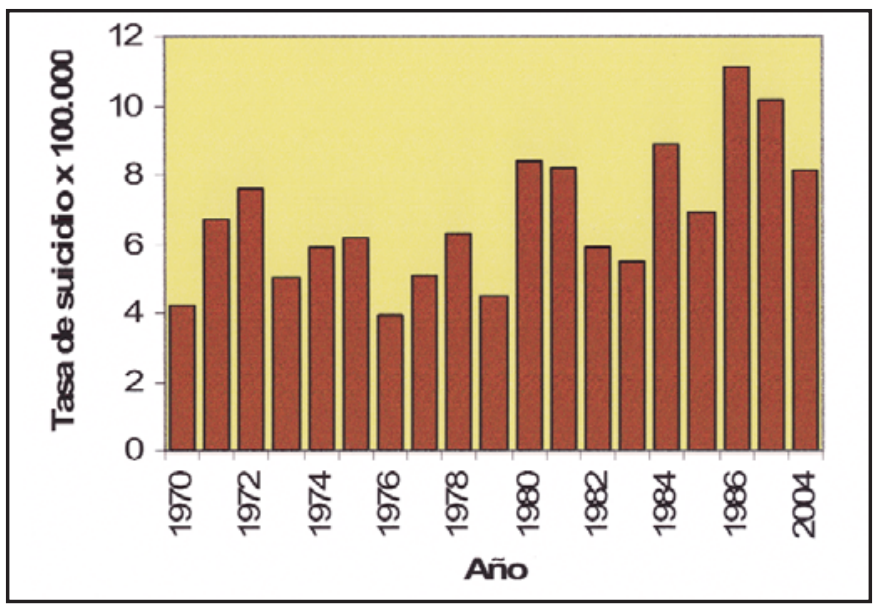


De 1953 a 1977 la edad media en varones fue de de 53,4 años y 52, I años en mujeres, pero en los últimos 10 años la edad media en las mujeres estaba acusando un importante descenso. Del 1978 al 1987 la edad media global fue de 51,9 años (50,2 años para los hombres y 53,8 años para las mujeres). La edad media global de los varones descendió respecto al periodo anterior y aumentó la de las mujeres. En 1989 la edad media de los hombres fue 41,6 y la de las mujeres de 52,6. En 2004 la media global de edad ha sido de 54,3 años (52, 7 para varones y 58,7 años para mujeres).

En relación al mecanismo utilizado, llaman la atención los cambios que se han producido a lo largo de los años en cuanto a las preferencias del suicida. En todos los periodos estudiados, y hablando en términos globales, la precipitación ha sido el más empleado. No obstante de 1953 a 1977 este mecanismo era seguido de la sumersión y el atropello por tren y de 1978 a 1987 por la ahorcadura y la ingesta de tóxicos. En 2004 se observa que el mecanismo de elección fue la ahorcadura (42,9\%) seguido de la precipitación (23,8\%) y del arma de fuego (7,5\%). La evolución de los principales mecanismos suicidas desde 1953 a 2004 se refleja en el gráfico 6.

\section{GRÁFICO 6}

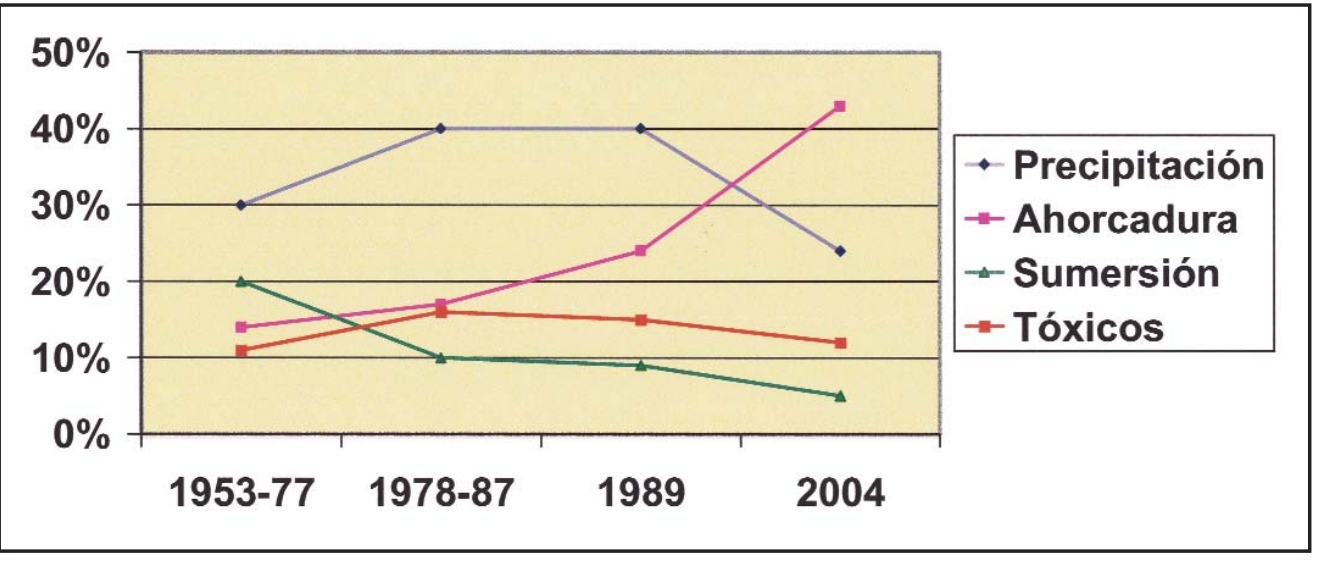

Tanto durante el periodo 1953-77 como 1978-87 las mujeres mostraron preferencia por la precipitación seguido de la ingesta de tóxicos. La sumersión fue el tercer mecanismo en frecuencia para el primer periodo y la ahorcadura para el segundo periodo. Estos datos no contradicen los recogidos para 2004 ya que la precipitación ha sido también en este caso, el medio más empleado.

Aunque en la tabla I consta en segundo lugar la ahorcadura, debe tenerse en cuenta que en el presente estudio se ha considerado la muerte por ingesta de cáusticos como un mecanismo distinto de la intoxicación mientras que en los estudios a que nos referimos se incluyen dentro de la muerte por ingesta de tóxicos. De esta forma los mecanismos más frecuentemente empleados por las mujeres para la ejecución del acto suicida coinciden cualitativamente en nuestro estudio y en los periodos anteriores (precipitación seguida de ingesta de tóxicos). La sumersión ha pasado de ocupar el tercer lugar en el primer periodo hasta el quinto lugar en el 2004.

En el caso de los hombres, la máxima frecuencia para la precipitación se registró de 1953 a 1977 seguida de la asfixia por sumersión en segundo lugar y ahorcadura y atropello por tren en tercer lugar. En el periodo 1978-87 la precipitación ocupó también el primer lugar, en este caso seguido de la ahorcadura y de la sumersión. El aumento en la frecuencia de suicidios por ahorcadura 
ha colocado a este mecanismo en el primer lugar en frecuencia seguido de la precipitación en el año 2004. El tercer mecanismo más empleado es el arma de fuego. La asfixia por sumersión ha pasado a ocupar el quinto lugar.

En relación a la época del año, aunque en los estudios anteriores se ha dividido el año en trimestres, de forma aproximada puede decirse que tanto en estos como en el que aquí se presenta el mayor número de suicidios se han registrado de abril a septiembre (primavera y verano).

Se desconocen los datos acerca de la existencia de cartas de despedida en los estudios realizados previamente en Sevilla. En 2004 sólo se encontraron documentos de despedida en el 8,84\% de los casos. Si comparamos con estudios realizados en otras regiones esta cifra no dista mucho del 8,7\% en que se encontraron estos documentos en el partido judicial de Cambados en el análisis realizado de 1989 a 1998 [8] o del I0,5\% que se refiere para los años 1972-1978 en L'Hospitalet [9]. Sin embargo en el partido judicial de Badajoz, en los casos de suicidio ocurridos de 1990 a 1995 el 35,29\% de los suicidas dejó documento de despedida [ [ 0].

En el $47,6 \%$ de los casos el cadáver fue encontrado en domicilio y en un $26,5 \%$ en vía pública. En el resto de los casos, el cadáver procedía del hospital, el centro de trabajo, instituciones penitenciarias o instituciones psiquiátricas. Como en otros estudios, parece que se mantiene la preferencia por el propio domicilio para la consumación del suicidio (Cambados $60,9 \%$ [8], Badajoz 57,5\% [10]) especialmente si tenemos en cuenta que algunos de los casos que se han consignado como procedentes de vía pública se trata de precipitaciones desde el propio domicilio por lo que probablemente el porcentaje de suicidios cometidos en domicilio sea mayor de lo que se refleja.

En cuanto al estado civil de los suicidas, el 31,2\% estaban casados, el 26,5\% solteros, el $14,3 \%$ eran viudos y un 6,1 \% estaban separados o divorciados. El orden de frecuencias del estado civil coincide con el registrado en Andalucía de forma global para el periodo de tiempo de 1976 a 1996 [I I].

Durante muchos siglos se ha tendido a interpretar el suicidio como una manifestación sintomática de enfermedad mental, especialmente de los trastornos depresivos y de la esquizofrenia [12]. En concordancia con esta afirmación, en Sevilla el 57, I\% de los sujetos tenía antecedentes patológicos de tipo psiquiátrico y el 7,5\% padecía enfermedad orgánica. El trastorno que con más frecuencia se registró fue la depresión seguido de la esquizofrenia. En un 10,7\% de los casos existían antecedentes de consumo de drogas de abuso.

En ocasiones, antes de la consumación del suicidio hay intentos previos o ideación autolítica. En un 82,3\% de los casos no se recogió expresamente este dato. Esto puede deberse a que no hubo intentos autolíticos previos ni ideación autolítica o a desconocimiento u ocultación por parte de los familiares. Un 8,2\% de los sujetos había verbalizado su intención suicida y el 9,5\% había hecho intentos previos. Las cifras de intentos autolíticos previos se encuentran por debajo de las registradas en los estudios de L'Hospitalet (I2\%) [9] y Cambados (17,4\%) [8].

Se practicaron análisis químico-toxicológicos en 115 casos $(78,2 \%)$ obteniéndose resultados negativos en el 30,4\% y positivos en el $69,4 \%$. Del mismo modo que en el estudio realizado en Cambados [8], en el que el alcohol etílico fue la sustancia más detectada (50\%), la sustancia que con más frecuencia se identificó fue el etanol de forma aislada (13,9\%) o asociado a otras sustancias como benzodiazepinas (6, I\%) o cocaína (3,5\%).

La existencia de antecedentes patológicos psiquiátricos concuerda con los resultados analíticos obtenidos, ya que detectaron benzodiazepinas en 25 casos (21,7\%), antidepresivos en 13 ( I 1,3\%) y neurolépticos en 3 (2,6\%). 


\section{CONCLUSIONES:}

I. En 2004 se realizaron en el SPF-IMLSE 919 autopsias de las cuales el 59\% fueron violentas. Los suicidios constituyeron el I5,9\% de las autopsias totales realizadas.

2. Desde los años 1950 los suicidios han pasado de representar el $14,8 \%$ de las muertes violentas hasta el 27, $12 \%$ registrado en 2004 .

3. La tasa de suicidio en Sevilla en 2004 ha sido de 8,36 por 100000 habitantes. Parece que existe cierta estabilidad en el número de suicidios desde mediados del siglo $X X$ en Sevilla tras la leve tendencia al aumento que se registró a finales de los años 80.

4. El suicidio continúa siendo más frecuente entre hombres que entre mujeres siendo la relación de 2,6:1.

5. La media global de edad fue de 54,3 años (52,7 años en varones y 58,7 años en mujeres). Para todos los periodos de tiempo estudiados la media de edad tanto para hombres como para mujeres se sitúa en la quinta década de la vida con la excepción del año 1989 en que se acusó una disminución en la edad media en varones.

6. Se ha producido un cambio en cuanto a la frecuencia de los mecanismos empleados para cometer el suicidio:

- En el partido judicial de Sevilla el mecanismo más frecuente de suicidio sigue siendo en el año 2004 la precipitación. En la provincia, el mecanismo más frecuente fue la ahorcadura. Siendo igualmente el mecanismo más utilizado en el global de la población.

- Desde 1953 los mecanismos más empleados por las mujeres han sido la precipitación y la ingesta de tóxicos. Desde 1978 el tercer lugar en frecuencia lo ha ocupado la ahorcadura. En 2004 se registró el mismo número de suicidios por ingesta de tóxicos que por ahorcadura, en mujeres.

- De 1953 a 1987 la precipitación ocupó el primer lugar como mecanismo de suicidio para varones. Sin embargo, el aumento progresivo en el empleo de la ahorcadura ha hecho que este mecanismo pasara de ocupar el tercer lugar para el primer periodo que se estudia, a ser el mecanismo más empleado en 2004.

- El uso de arma de fuego y el suicidio a lo bonzo han sido casi exclusivos del sexo masculino en 2004.

7. En todos los periodos, incluido en 2004 , el mayor número de suicidios se ha registrado en el segundo y tercer trimestre del año (de abril a septiembre).

8. En 2004 sólo hay constancia de la existencia de notas suicidas en un 8,84\% de los casos. Esta cifra es similar a la recogida en estudios realizados en otras regiones españolas.

9. El domicilio sigue siendo el lugar de preferencia para la consumación del acto suicida y es el lugar donde se encuentra el cadáver en el 47,6\% de los casos durante el año 2004.

I0. Tanto en Sevilla durante el año 2004 como en la Comunidad Autónoma de Andalucía el orden de frecuencia del estado civil es de forma global casado, soltero, viudo y separado o divorciado. 
II. De los I 5 casos en que se practicaron análisis toxicológicos, el alcohol etílico fue la sustancia más detectada, seguido de las benzodiazepinas y los antidepresivos.

I2. En el 57, I\% de los suicidas en Sevilla durante el 2004 había antecedentes patológicos de tipo psiquiátrico, siendo los cuadros más frecuentes la depresión, la esquizofrenia y el consumo de drogas de abuso. Este dato concuerda con los resultados toxicológicos obtenidos, ya que se detectaron benzodiazepinas en un $21,7 \%$ de los casos, antidepresivos en un I I,3\% y neurolépticos en un 2,6\%.

\section{AGRADECIMIENTOS:}

A Maite Barba, bibliotecaria del INTCF de Sevilla, por su valiosa ayuda en la búsqueda bibliográfica. A todo el personal del SPF-IMLSE por su inestimable colaboración en la recogida de los datos del estudio.

\section{BIBLIOGRAFÍA:}

I.- Comunicado de prensa de la Organización Panamericana de la Salud (OPS), (9 sept. 2005). www.who.int/mediacentre/news/releases/2004/pr6l/es/

2.- Estadísticas del suicidio en España en 2003. Instituto Nacional de Estadística. http://www.ine.es/inebase/cgi/axi

3.-Informe de la SESPAS 1999. www.sespas.es/informe2000/dI I2.pdf

4.- Heredia F. El suicidio en Sevilla en 1989. Estudio epidemiológico.

Rev Esp Med Legal 1991;66/67,68/69:57-60.

5.- Romero Palanco JL. Aspectos epidemiológicos del suicidio en la ciudad de Sevilla referidos a los años 1953 a 1977. Rev Esp Med. Legal 1985;42/43:35-54.

6.- Romero Palanco JL, Gamero Lucas J, Vizcaya Rojas MA, Arufe Martínez M. I, Hernández Treviño A. Evolución del suicidio consumado en la ciudad de Sevilla en los años 1978 a 1987. Rev Esp Med Legal 1989;58/59:9-2I.

7.- Heredia F. Estudio del suicidio de adolescentes en Sevilla en 1989. Rev Esp Med Legal 1991;66-67,68-69:61-64.
8.- Dorado Fernández E, Rodes Lloret F. Suicidio por ahorcadura en el Partido Judicial de Cambados (1989-1998). Estudio epidemiológico y comparativo. Cuad Med Forense 2000;19:9-19.

9.- Camps Surroca D, Puig Bausili L, Pujol Robinat A. Estudio del suicidio consumado en el Partido Judicial de L'Hospitalet (años 1972-1978). Rev Esp Med Legal 1980;24-25: 72-77.

10.- Casado Blanco M, Sánchez Ugena F, Hernández Gil ML, Merino Zamora MJ. Análisis del suicidio consumado en el partido judicial de Badajoz durante el periodo 1990-1995. Cuad Med Forense 1998;13:77-83.

II.- Ruiz Ramos M, Muñoz Bellerín J, Ramos León J.M, Gil Arrones J, Ruiz Pérez I, Muriel Fernández R. Tendencia a la mortalidad por suicidio en Andalucía desde 1976 a 1995. Gaceta Sanitaria 1999;13(2):I35-140.

12.- Corbella Corbella J: El suicidio. En: Gisbert Calabuig J.A. Medicina Legal y Toxicología. $6^{a}$ edición. Editado por Masson S.A. Barcelona 2004.pp 322-330. 